\title{
A Neuro Based Level Irregularity to Efficient Acknowledgment of Variations in Online Social Networks
}

\author{
S Divya, M Ravi, V Akhila, G Sneha, K Shilpa
}

\begin{abstract}
Use of casual association is the principal handiness of the present life. With the happening to a regularly expanding number of online electronic life, the information open and its use have gone under the danger of a couple of irregularities. Variations from the norm are the huge purpose behind online fakes which license information access by unapproved customers similarly as information delivering. One of the variations from the norm that go about as a tranquil attacker is the level eccentricity. These are the anomalies realized by a customer because of his/her variable direct towards different sources. Level inconsistencies are difficult to recognize and risky for any framework. In this paper, a self-recovering neuro-soft philosophy (NHAD) is used for the disclosure, recovery, and removal of level irregularities efficiently and unequivocally. The proposed philosophy works over the five models, specifically, missing associations, reputation gain, significant refinement, trust properties, and trust score. The proposed approach is evaluated with three datasets: DARPA'98 benchmark dataset, designed dataset and consistent traffic. Results show that the precision of the proposed NHAD show for $10 \%$ to $30 \%$ idiosyncrasies in made dataset goes some place in the scope of $98.08 \%$ and $99.88 \%$. The evaluation over DARPA'98 dataset demonstrates that the proposed procedure is better than anything the present courses of action as it gives $99.97 \%$ recognizable proof rate to odd class. For ceaseless traffic, the proposed NHAD exhibit works with an ordinary accuracy of $99.42 \%$ at $99.90 \%$ recognizable proof rate.
\end{abstract}

Keywords: Horizontal Anomaly, Social Networks, Reputation, Neuro-Fuzzy Model.

\section{INTRODUCTION}

Online relational associations grant efficient correspondence between the customers and the information sources. With the methodology of continuously online web based life, controlling the information get to has transformed into a significant test. One of these errands incorporates identification of framework anomalies and special cases. Peculiarities are the abrupt lead of the customer which results in sporadic and suspicious activity making risks the ordinary framework customers. In perspective on the framework, these eccentricities empower customer information to be recouped without assent and use it against the capacity of online system. Quirks are ordered into static named, static unlabeled, amazing checked and dynamic unlabeled. Framework peculiarities impact the utility similarly as the relationship

Revised Version Manuscript Received on 16 September, 2019.

* Correspondence Author

S DIVYA*, CSE Department, VJIT, Hyderabad, India. Email: divyas@vjit.ac.in

M RAVI, MCA Department, JBIET, Hyderabad, India. Email: ravimjbiet@gmail.com

V AKHILA, G SNEHA, K SHILPA (PG Scholars) MCA Department, JBIET, Hyderabad, India. between the systems and customers. Irregularity disclosure can be performed in different unmistakable ways: classification (oversaw approach), gathering, apparition examination, information theoretic, nearest neighbor and quantifiable systems. With the presence of extended online social affiliation goals, customer following and irregularity acknowledgment in casual associations are two of the genuine zones of research. The fundamental goal of recognizing abnormalities is to perceive the adjusted examples of attentive activities in the framework. A huge amount of research has been done to make a summed up method for a nomaly detection. Several well-made procedures are available for remembering them under specific conditions on different territories. A champion among the most threatful irregularities prevalent in the online relational association is the level variation from the norm. Indeed, even inconsistency is one of a kind in connection to static and dynamic classification and has a spot with the direct classification of social variations from the norm. It implies the qualification in the association direct of the customer reliant on the customers' particular development in a system over the online casual network. Level. characteristic is difficult to pursue and recognize as it thoroughly depends upon the unmistakable sources worked together by a customer. A customer may encounter explicit lead towards a particular source which could be treated as an eccentricity. Accordingly, it winds up most outrageous basic to meticulously portray the complete system which can speedily recognize the suspicious direct and can resolve these anomalies. Over the span of the latest couple of years, disclosure of the peculiarities has been taken as a certified research which required efficient approaches for improved identification. In any case, the philosophies proposed so far are authentic for frameworks under certain pre-defined parameters which generally incorporates the component of information exchange between the source and the customers. The present composition needs to give an all out response for the even peculiarity issue in the online casual networks regardless of the component of peril it may cause. Further, there is no structure of the parameters which can be utilized for the area of level abnormalities. The present courses of action can resolve the variations from the norm using the framework activity rather than the customers' approach towards a particular source. Evaluations performed dependent on framework activity can give wrong results as customers' framework activity can be intentional or incidental, while the customers' steady association with a particular source can give more bits of knowledge in regards to its lead in online casual networks.

Courses of action like COPRA and Bayesian peculiarity revelation are

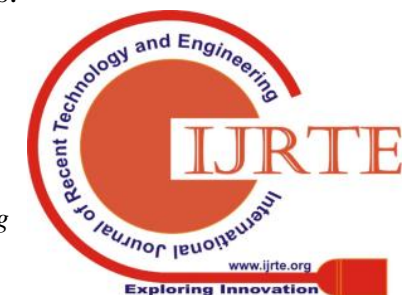


available for the distinguishing proof of irregularities in online casual associations. The Bayesian system utilizes the Bayesian filtering instrument to recognize the peculiar center in the casual network, however COPRA deals with the identification of the overlapping communities in the relational associations. COPRA can be used to perceive inconsistencies by choosing the customers in the non-covering systems. Regardless of the way that these strategies are ground-breaking, they are unfit to give recovery

and slaughter instruments. Existing neuro-cushy strategies like Mobile Fuzzy Trust Inference, Modularity intensification and Hybrid Genetic Detection can in like manner be connected for recognizing various customers in a given relational association. In any case, at present, these procedures are evaluated for perceiving trust between two customers and for system revelation. On an increasingly broad variation, these techniques can be consolidated with irregularity distinguishing proof framework and their present correspondence classification can be used for perceiving even quirks. In any case, this may manufacture the flightiness of the general system. Some extraordinary plans fuse co-bundling based total irregularity revelation using framework models, and self-learning interference acknowledgment structures that use Radial Basis Functions (RBF) neural framework to decide abnormalities. Moreover, there are various techniques that basically base on passing on Support Vector Machine (SVM) close by various conviction frameworks to recognize odd direct. A bit of these are peculiarity acknowledgment with indispensable part analysis and SVM, autonomous labeling with SVM, and outfit framework for irregularity ID which uses SVM in mix with the Extended Kalman Filter. Disregarding the way that, the execution delayed consequences of these game plans over standard benchmarks prescribe their efficiency, yet these don't contain fitting features of online casual networks which are required for the detection of horizontal anomalies. Efficient strategies are required which cannot only target the identification of even characteristic as an issue yet should be fit for recovering the whole framework proficiently with high exactness. Subsequently, the objectives of this paper consolidate the identification of level anomalies, recovery of customers and transfer of non-recoverable customers eating up less cycles with lower botches, higher accuracy and less dissatisfactions. In this paper, a neuro-feathery based level anomaly area (NHAD) shows is recommended that grants revelation, recovery, and clearing of level variations from the norm productively and precisely .NHAD works over five ideal models, to be specific: missing associations, reputation gain, significant differentiate, trust properties, and trust score. Directly off the bat, the model structures the trust-based reputation graph. It by then develops oneself patching neural model reliant on its trust properties. Next, it uses the cushioned deducing system to finalize the final cost, in perspective on which a decision is made inside seeing the peculiarity. The proposed NHAD show grants proficient and exact acknowledgment of level variations from the norm in online relational associations.

\section{RELATED WORKS}

The variation from the norm acknowledgment in online relational associations can be finished in different ways. Consistently, various variations Of Anomalies have been identified and focused with key courses of action. These game plans revolve around the request of the idiosyncrasy and a short time later give game plans which can resolve the issue of customer identification.

\section{A. Vehicular and crowd anomaly detection:}

The element of abnormalities can influence the utility of the casual association and this has been considered as the vehicular irregularities by Giridhar et al. under the name of ClariSense. The makers proposed an increase to the anomaly illumination system and attempted their strategy in the vehicular condition. Their strategy revolves around the sensor limits of the framework and identifies the issues related to the occasion of the variations from the norm in the tantamount condition. Chaker et al. considered the gathering irregularity revelation and confinement in both adjacent and worldwide casual networks. Pleasant components are used by the makers to recognize the gathering peculiarity with higher precision.

\section{B. Rule based anomaly detection:}

Defining the guidelines for coordinated effort can help in identification of peculiarities. Akoglu et al. considered the irregularities in the weighted diagrams and developed an Oddball count for finding the impacted centers. The makers utilized the rule based approach to manage recognize these graph variations from the norm. The above philosophies are prepared for perceiving a particular irregularity in a compelled circumstance. These philosophies are not prepared to recognize center point lead in online relational associations as these depend just on the relationship between the center points, which can be controlled adequately. This control can be the result of different properties for different affiliations.

\section{Exchanged off Account-based Anomaly Detection:}

Another piece of the abnormalities in the online casual associations is the bartered records which have been broke down by Egele et al.. The makers developed a procedure under the name of COMPA, which can perceive the dealt records in most of the individual to individual correspondence regions. The makers separated and attempted their technique on a sweeping enlightening list including around 1.4 billion Twitter messages which are freely accessible. These frameworks can be classified as Intrusion Detection Systems (IDS) particularly focusing on the anomaly revelation in the online informal communities as expressed by Sommer and Paxson. The authors showed the utility of AI approaches to manage the plan of an IDS which can effectively pursue the framework irregularities. These variations from the norm are compelled to the social records, yet these can moreover have opposing effects the frameworks working these vindictive sources. Zhu et al. considered The Similar Aspect Of The Anomalies in The cell frameworks. The makers utilized the online life traffic and the phone data for worm guideline in cell frameworks. Identification of exchanged off records is one of the genuine troubles and the above philosophies are fitting. Nevertheless, these approachs can be used after a strike. Computer based intelligence parts are efficient, 
yet in the above cases, a pre-vital planning of the acknowledgment system is required, which can be avoided by an irregular center point.

\section{Coordinated effort based Anomaly Detection:}

Motivation behind joint effort can be another response for recognizing variations from the norm. Such procedure utilizes the possibility of quirk scores by separating the sources with which a customer interfaces. Takahashi proposed change-point acknowledgment technique which uses the Sequentially Discounting Normalized Maximum Likelihood (SDNML). The makers utilized the quirk scores gained from these examinations to recognize the association abnormalities. In the other strategy by $\mathrm{Yu}$ et al., the makers proposed a Group Latent Anomaly Detection (GLAD)approach which uses the pair-wise as well as pointwise data to derivation at the final selection of inconsistencies. Their procedure is efficient yet needs pertinence to the dimension peculiarities considering its dependence on social event features for each individual, however even anomalies a rise due to an individual's activity irrespective of the group to which it has a spot.

\section{E. True Anomaly Detection:}

Bits of knowledge can be another response for the issues related to the peculiarity recognizable proof. Using the possibility of estimations, Heard et al. Proposed an efficient system for anomaly revelation in the relational associations, which particularly uses the Bayesian examination approach. A two-organize approach is used by the makers for the abnormality revelation which diminishes the get-together of possibly peculiar center points. The present courses of action depend much on the assembled data, which can be used just by virtue of taught peculiarities. Regardless, realtime identification, checking and forewarning systems are prohibited in the present philosophies, which are required for the course of action of an efficient structure for recognizing level variations from the norm. Past work showed in this portion clearly exhibits that most of the present procedures have been customary in the disclosure of the irregularities and have not pondered the level abnormalities. As such, efficient approaches are required which can not simply recognize the peril level achieved by those anomalies yet moreover settle these efficiently.

\section{EXISTING SYSTEM}

The present game plans can resolve the irregularities using the framework activity instead of the customers' system towards a source. Evaluations performed dependent on framework activity can give mixed up results as customers' framework development can be conscious or inadvertent, while the customers' perpetual relationship with a particular source can give more bits of knowledge in regards to its direct in online relational associations. Plans like COPRA and Bayesian peculiarity distinguishing proof are available for the area of characteristics in online casual networks. The Bayesian approach utilizes the Bayesian isolating part to perceive the bizarre center point in the relational association, while COPRA deals with the distinctive verification of the covering systems in the casual networks. COPRA can be used to recognize inconsistencies by choosing the customers in the non-covering systems. In spite of the way that these approachs are fruitful, they are unfit to give recovery and execute instruments. Existing neuro-fleecy systems like Mobile Fuzzy Trust Inference, Modularity development and Hybrid Genetic Detection can in like manner be connected for distinguishing various customers in a given casual association. At present, these philosophies are surveyed for perceiving trust between two customers and for system disclosure. On a progressively broad structure, these procedures can be composed with irregularity acknowledgment instrument and their present correspondence gathering can be used for recognizing level variations from the norm. Regardless, this may assemble the multifaceted idea of the general system. Some unique game plans fuse co-clustering based total anomaly revelation using framework precedents, and self-learning intrusion acknowledgment structures that usage Radial Basis Functions (RBF) neural framework to decide irregularities. In like manner, there are various strategies that basically revolve around sending Support Vector Machine (SVM) nearby unique conviction frameworks to recognize peculiar lead. A segment of these are irregularity disclosure with boss part examination and SVM, independent stamping with SVM, and troupe system for anomaly acknowledgment which uses SVM in mix with the Extended Kalman Filter. Regardless of the way that, the execution results of these courses of action over standard benchmarks suggest their viability, yet these don't contain reasonable features of online casual associations which are required for the distinguishing proof of level anomalies.

This journal uses double-blind review process, which means that both the reviewer (s) and author (s) identities concealed from the reviewers, and vice versa, throughout the review process. All submitted manuscripts are reviewed by three reviewer one from India and rest two from overseas. There should be proper comments of the reviewers for the purpose of acceptance/ rejection. There should be minimum 01 to 02 week time window for it.

\section{PROPOSED SYSTEM}

The proposed NHAD show goes for denoting a particular customer in a system of an online relational association to be a characteristic or not. NHAD uses the present self-recovering neural model for instating the peculiarity recognized as a deceptive neuron in the neural setup of the systems of an online casual association. By then, this neural model recovers using a feathery induction structure with possible results of recovering a customer before absolutely destroying it. The reputation increment of each customer goes about as a weight, and a patching cost is enrolled for all of the customers. This recovering expense is then used to find a definitive outcome for a center point's activity; for instance either an irregularity or a credible customer. For recovering model application, the model is arranged as the neural setup showed up in Fig. 3. The neural setup speaks to the m number of customers in the jth organize each treated as a data neuron with weight indistinguishable to their reputation gain. The covered layer ("sources" in Fig. 3) is confined from the sources reliant on the customer activity. The yield of the neural model makes an edge cost underneath which the customer is treated as a characteristic. The last cost of a customer is resolved after Defuzzification of the soft set over Tp. 


\section{ARCHITECTURE}

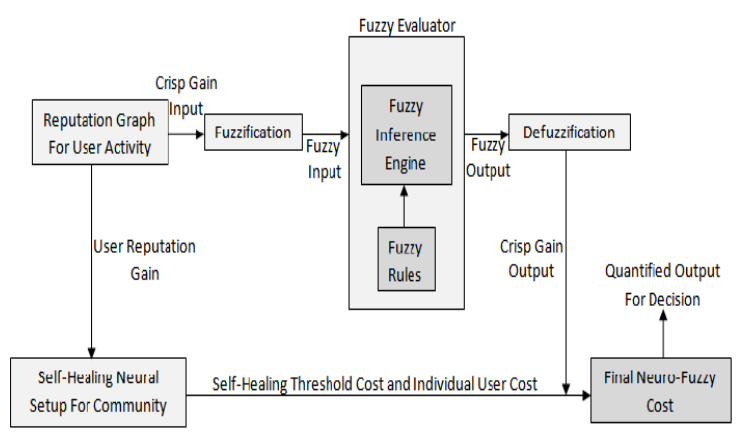

Fig 1: Architecture of the system

\section{A. Reputation outline:}

The proposed system outlines the reputation graph and after that uses the fleecy structure to evaluate each customer over the considered properties for their activities in a casual association. Next, this reputation graph is used to find oneself recovering expense of each customer. Following this, the edge recovering cost, solitary cost and crisp consequences of each customer are used to find the last neuro-cushioned cost, which is used to pick whether a customer is an irregularity or not.

\section{B. Repairing Cost and Neuro-Fuzzy Formations:}

The underlying stage in the proposed NHAD show is to portray described arrangement of properties to the neural framework which works by using a patching cost. The mapped framework is then taken a shot at the cushy enlistment standards to create the feathery sets for the lead of each center, which is then surveyed to get in contact at a decision of announcing a center point as a quirk or not.

\section{Tremendous Difference:}

It relies upon the case of joint effort between the two components, and it helps in recognizing verification of a customer as a peculiarity. Vital difference controls the customers' reputation increment and its development over the online life. The vital complexity is extraordinarily impacted by the customer development over unverified sources. In this paper, the institutionalized controlling edge deviation of a customer in a system is fixed at an edge of 0.5. This regard is fixed reasoning about that at the most a framework can have half eccentricities. Regardless of the way that in a certified framework, this regard is uncommonly low, yet to show the feasibility of the proposed strategy, a higher anomaly rate is picked.

\section{Reputation Gain:}

Reputation gain $\mathrm{Rg}$ is handled over a graph $\mathrm{G}$ with the ultimate objective that $\mathrm{G}=(\mathrm{Tp}$; $\mathrm{Ts})$, where $\mathrm{Tp}$ implies the course of action of trust properties that structure the vertices of the chart, and Ts is the plan of trust score assigned as weight to the edges interfacing the vertices (Trust Properties) to a particular customer.

\section{RESULTS OVER SYNTHETIC DATA}

This section provides the details of proposed NHAD model. The results over the synthetic data as part of the analysis are as follows:

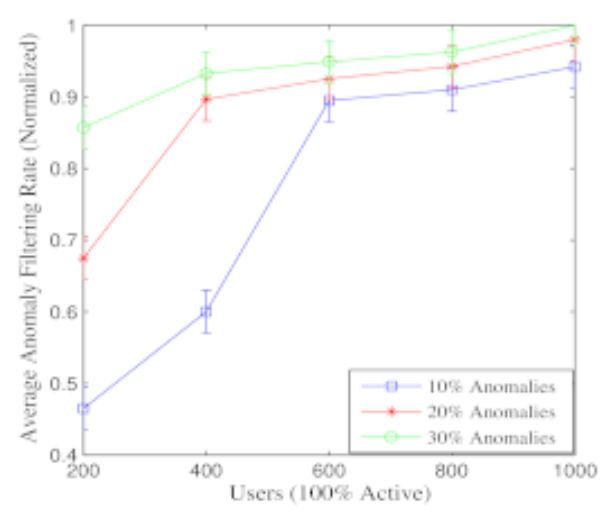

Fig 2. Average anomaly filtering rate vs. total active users

This is to analyze for anomaly filtering rate with percentage of active users. The proposed NHAD model gives better filtering rate as it takes little iteration to identify an anomaly.

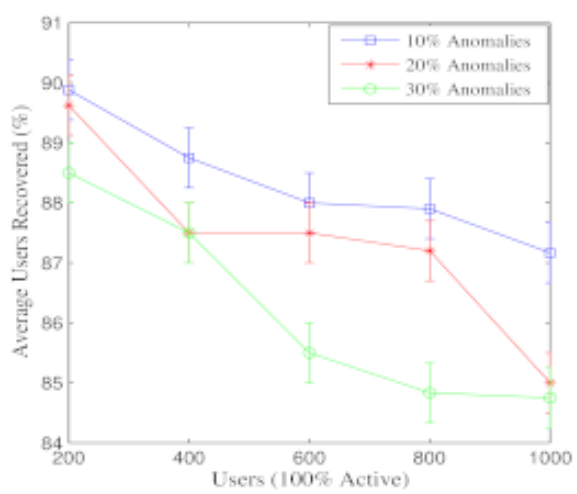

Fig 3. Average users recovered(\%) vs. total users

The proposed NHAD model analyze the percentage of users recovered is compared with the previous analysis i.e average anamoly filtering rate. The average percentage of users recovered during analysis as $88.4 \%, 87.4 \%, 86.3 \%$ for $10 \%$, $20 \%$ and $30 \%$ anamolies respectively.

\section{CONCLUSION}

In this paper, a neuro-cushy based level irregularity area (NHAD) show is prescribed that speaks to efficient acknowledgment of even variations from the norm in online social networks. The proposed NHAD model uses self-healing neural exhibit and a fleecy inference system with the probability of recovering a customer before executing it. The proposed philosophy was surveyed in three segments. The first surveyed the proposed NHAD show using a DARPA'98 dataset as used by most of the twofold classification courses of action, the second part evaluated it using produced dataset and the third part evaluated the proposed model over continuous traffic. The retouching cost system of the proposed NHAD show allowed acknowledgment, recovery and clearing decisions in less emphasess, thusly, making it an efficient plot for the disclosure of even variations from the norm in online relational associations. The proposed approach uses limited plans despite for recognizing complex level variations from the norm and the amount of cycles required for getting in contact at a decision isn't actually the theoretical characteristics. Further, the mapping of the trust properties 
into the final yield should be conceivable in an unfaltering time. In any case, the principle multifaceted nature included is the basic mapping of cushioned guidelines and required yield. Currently, these rely upon exploratory appraisals yet can be advertisement libbed through learning over oneself recovering neural model. Another genuine good position is in the inside suggestion of the proposed procedure, which is relied on the efficient recovery part of its base neural model. With less cycle to settle, the neural model support efficient association of the proposed approach. The examination in the paper shows that the proposed philosophy can be used as an offline approach for perceiving eccentricities out of dataset similarly as an online procedure for recognizing anomalies at the continuous. Results prescribe that the proposed NHAD show ends up being efficient to the extent significant increments achieved in relationship with the present philosophies over various parameters to be explicit, quirk filtering rate, accuracy in peculiarity recognizable proof, blend regard, approach dissatisfactions, and the dimension of customers recovered notwithstanding being an anomaly.

\section{REFERENCES}

1. T.-H. Tsai, W.-C. Jhou, W.-H. Cheng, M.-C. Hu, I.-C. Shen, T. Lim, K.-L. Hua, A. Ghoneim, M. A. Hossain, and S. C. Hidayati, "Photo sundial: estimating the time of capture in consumer photos," Neurocomputing, vol. 177, pp. 529-542, 2016.

2. B. Wu, W.-H. Cheng, Y. Zhang, and T. Mei, "Time matters: Multiscale temporalization of social media popularity," in Proceedings of the 2016 ACM on Multimedia Conference, pp. 1336-1344, 2016.

3. C.-W. You, Y.-L. Chen, W.-H. Cheng, M.-S. Chen, and S.-A. Tsai, "Socialcrc: a social-and context-aware rendezvous coordination system," in CHI'10 Extended Abstracts on Human Factors in Computing Systems, pp. 3391-3396, 2010.

4. D. Savage, X. Zhang, X. Yu, P. Chou, and Q. Wang, "Anomaly detection in online social networks," Social Networks, vol. 39, pp. 62-70, 2014.

5. Y. Liu and S. Chawla, "Social media anomaly detection: Challenges and solutions," in Proceedings of the 21th ACM SIGKDD International Conference on Knowledge Discovery and Data Mining, pp. 2317-2318, 2015.

6. M. Ahmed, A. N. Mahmood, and J. Hu, "A survey of network anomaly detection techniques," Journal of Network and Computer Applications, vol. 60, pp. 19-31, 2016.

7. H. Ko, S. Pack, and W. Lee, "Timer-based push scheme for online social networking services in wireless networks," IEEE Communications Letters, vol. 16, no. 12, pp. 2095-2098, 2012.

8. J. Gao, N. Du, W. Fan, D. Turaga, S. Parthasarathy, and J. Han, "A multi-graph spectral framework for mining multi-source anomalies," in Graph Embedding for Pattern Analysis, pp. 205-227, Springer, 2013.

9. S. Gregory, "Finding overlapping communities in networks by label propagation," New Journal of Physics, vol. 12, no. 10, pp. 1-26, 2010.

10. N. A. Heard, D. J. Weston, K. Platanioti, D. J.Hand, et al., "Bayesian anomaly detection methods for social networks," The Annals of Applied Statistics, vol. 4, no. 2, pp. 645-662, 2010.

11. F. Hao, G. Min, M. Lin, C. Luo, and L. T. Yang, "Mobifuzzytrust: an efficient fuzzy trust inference mechanism in mobile social networks," IEEE Transactions on Parallel and Distributed Systems, vol. 25, no. 11, pp. 2944-2955, 2014.

12. J. Su and T. C. Havens, "Quadratic program-basedmodularity maximization for fuzzy community detection in social networks," IEEE Transactions on Fuzzy Systems, vol. 23, no. 5, pp. 1356-1371, 2015.

13. J. Su and T. C. Havens, "Fuzzy community detection in social networks using a genetic algortihm," in Fuzzy Systems (FUZZIEEE), 2014 IEEE International Conference on, pp. 2039-2046, 2014.

14. X. Zhng, B. Zhang, C. Zhang, and A. Ma, "A multi-objective hybrid genetic algorithm for detecting communities in complex networks," in Natural Computation, Fuzzy Systems and Knowledge Discovery (ICNC-FSKD), 2016

\section{AUTHORS PROFILE}

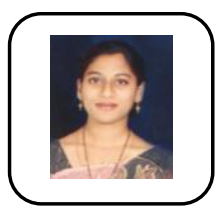

S DIVYA, completed B.Tech (CSE) in 2009 with First class and Distinction, M.Tech(CSE) in 2012 with First class and Distinction. working as Assistant Professor in CSE Department, VJIT,Hyderabad,

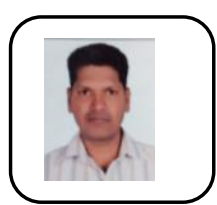

M RAVI, completed MCA in 2009, M.Tech(CSE) in 2011. currently working as Assistant Professor in MCA Department, JBIET, Hyderabad,

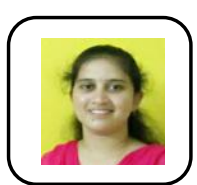

V AKHILA, PG Scholar, MCA Department JB Institute of Engineering \& Technology, Hyderabad.

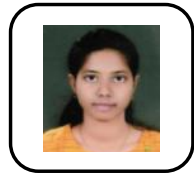

G SNEHA, PG Scholar, MCA Department JB Institute of Engineering \& Technology, Hyderabad words.

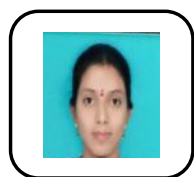

K SHILPA Assistant professor, MCA Department, JB Institute of Engineering \& Technology, Hyderabad. 